Published by Al-Nahrain College of Medicine P-ISSN 1681-6579

E-ISSN 2224-4719

Email: iraqijms@colmed-alnahrain.edu.iq

http://www.colmed-alnahrain.edu.iq

http://www.iraqijms.net

Iraqi JMS 2018; Vol. 16(3)

\title{
Pre and Post Extracorporeal Shock Wave Lithotripsy (ESWL) Urine Culture as A Guide for Antibiotics Management
}

\author{
Laith A. Al-Anbary ${ }^{1} A B U, F I C M S$, Ahmed A. Khaleel ${ }^{2} M B C h B$, Jabbar S. Hassan ${ }^{3} P h D$ \\ ${ }^{1}$ Dept. of Surgery, College of Medicine, Al-Nahrain University, Baghdad, Iraq, ${ }^{2} \mathrm{Al}$-Imamein Al-Kadhimein Medical City, \\ ${ }^{3}$ Dept. of Medical Microbiology, College of Medicine, Al-Nahrain University, Baghdad, Iraq
}

\begin{abstract}
Background
\end{abstract}

The lifetime prevalence of kidney stone disease is estimated at $1-15 \%$. The positive urine cultures can be obtained not only from Struvite stones, but also from calcium oxalate stones and also high levels of endotoxins are found both in infection stones (Struvite and carbonate apatite stones), and in non-infection stones. High concentrations of endotoxins (lipopolysaccharides) are thought to be released in the systemic circulation during stone treatment, inducing a systemic inflammatory response (SIRS) and this leads to urosepsis. PreExtracorporeal shock wave lithotripsy (ESWL) prophylactic antibiotics have an important role in reducing postSWL infections, however, previous studies reported conflicting results. The issue of administering prophylactic antibiotics remains controversial in patients with sterile urine undergoing ESWL.

$\begin{array}{ll}\text { Objective } & \text { To evaluate possible risk factors for post ESWL bacteriuria and consequently to identify patients with higher } \\ \text { danger for urinary tract infection (UTI) or sepsis. } \\ \text { Urine samples from } 50 \text { patients underwent ESWL, were collected by clean catch mid-stream urine collection } \\ \text { method in sterile containers. Those patients were attending and admitting to Al-Imamein Al-Kadhimein } \\ \text { Medical City during the period from October } 2016 \text { to January 2017. All patients had a urine culture performed } \\ \text { before and after shock wave lithotripsy. Statistical analysis was performed with Epi-Info } 7 \text { and Excel programs. } \\ \text { Statistical significance was evaluated using the Fisher's exact test with } p<0.05 \text { considered statistically } \\ \text { significant. }\end{array}$

Results A total of 50 patients who underwent ESWL during; the 2- months study period was enrolled in the study. Thirty-three $33(66 \%)$ out of 50 were men and $17(34 \%)$ out of 50 were women. $10(20 \%)$ of patients had hypertension and $5(10 \%)$ had diabetes mellitus. Fifty urine samples were collected from patients enrolled in this were cultivated on blood and MacConkey agar Pre-and post-ESWL Regarding Pre-ESWL results revealed 14 (28\%) were urine culture positive while 23 cases were post-SWL urine culture positive.

Conclusion Antibiotic prophylaxis is not justified without defined risk factors such as positive urine culture before ESWL, an external bladder catheter or nephrostomy tube and a history of infectious stones or recurrent urinary tract infection.

Keywords Extracorporeal shock wave lithotripsy (ESWL), urine culture, renal stone and antibiotics

Citation

Al-Anbary LA, Khaleel AA, Hassan JS. Pre and post extracorporeal shock wave lithotripsy (ESWL) urine culture as a guide for antibiotics management. Iraqi JMS. 2018; 16(3): 289-297. doi: $10.22578 /$ IJMS.16.3.8

List of abbreviations: AUA = American Urological Association, $\mathrm{EAU}=$ European Association of Urology, ESWL = Extra Corporal Shockwave Lithotripsy

\section{Introduction}

The lifetime prevalence of kidney stone disease is estimated at $1 \%$ to $15 \%$, varying according to age, gender, race, and geographic Location ${ }^{(1)}$. The most common component of urinary calculi is calcium, which is a major constituent of nearly $80 \%$ of stones. Calcium oxalate comprises about $60 \%$ of all stones; mixed calcium oxalate and hydroxyapatite make up $20 \%$ and brushite stones make up $2 \%$. Uric acid and Struvite (magnesium ammonium phosphate), each 
comprise approximately $7 \%$ of stones, and cysteine stones represent only about $1 \%$ (2). Infection stones are composed primarily of magnesium ammonium phosphate hexahydrate (MAP) $\left(\mathrm{MgNH}_{4} \mathrm{PO}_{4} \cdot 6 \mathrm{H}_{2} \mathrm{O}\right)$ (3). Struvite stones are infection stones frequently present as renal staghorn calculus. The driving force behind Struvite stone is infection of the urine with urease producing bacteria, the most common bacteria associated with stones are Proteus, Pseudomonas, Providencia, Klebsiella, Staphylococci, and Mycoplasma. The high ammonium concentration derived from the urea-splitting organisms results in an alkaline urinary $\mathrm{pH}$. The urinary $\mathrm{pH}$ of a patient with a MAP calculus rarely is $<7.2$ (normal urinary $\mathrm{pH}$ is 5.85). It is only at this elevated urinary $\mathrm{pH}$ (>7.19) MAP crystals precipitate. MAP crystals are soluble in the normal urinary $\mathrm{pH}$ range of 5$7{ }^{(4)}$. Infection stones occur most commonly in those prone to frequent urinary tract infections (UTI), Struvite stones occur more often in women than men by a ratio of 2:1 (5). The presence of urease-producing organisms in only $48 \%$ of Struvite stones, while $32 \%$ of calcium oxalate stones were infected. It may therefore be inferred that an infection from urease-producing organisms is not always present in infected Struvite stones; furthermore, positive cultures can be obtained not only from Struvite stones, but also from calcium oxalate stones ${ }^{(6)}$. Nano bacteria have also been suggested to cause stone disease and be pathogenic for Urosepsis following kidney stone treatment. Nano bacteria are microorganisms that are (10-100) times smaller than normal bacteria; they may be involved in the formation of calcium phosphate crystals, thus creating nidus for the formation of the stone. The risk of sepsis would therefore be correlated with the release of these microorganisms from the stone during treatment. Endotoxins are another factor supposed to be involved in the pathogenesis of urinary infection from kidney stones. High levels of endotoxins are found both in infection stones (Struvite and carbonate apatite stones), and in non-infection stones. High concentrations of these (lipopolysaccharides) are thought to be released in the systemic circulation during stone treatment, inducing a systemic inflammatory response (SIRS) and this led to urosepsis (7). Sepsis, SIRS and infection either documented or strongly suspected characterized by fever $\left(>38^{\circ} \mathrm{C}\right)$ or hypothermia $\left(<36{ }^{\circ} \mathrm{C}\right)$. Tachycardia $(>90$ beats/min in patients not on B-blockers), tachypnea (respiration $>20$ breaths $/$ min or $\mathrm{PaCO}_{2}<4.3 \mathrm{kPa}$ or a requirement for mechanical ventilation) White cell count $>12000$ cells $/ \mathrm{mm}^{3}{ }^{\text {(8) }}$. The primary predictive risk factors of urosepsis are the following: patient conditions such as immunodepression and a deteriorated performance status, a urinary infection in progress or a history of recurrent infections, characteristics of the stone, anatomy of the urinary tract. In the hospital setting, the most common causes are the presence or manipulation of indwelling urinary catheters, urinary tract surgery (PCNL), and urinary tract obstruction (particularly that due to stones obstructing the ureter) ${ }^{(9)}$. Urine cultures are performed if there is a suspicion of infectionrelated calculi or if there are signs or symptoms of a UTI. A culture that is positive for ureasplitting organisms such as Proteus, Pseudomonas, Klebsiella, Staphylococcus aureus, and Staphylococcus epidermidis would help explain the formation of a Struvite calculus. A positive culture also will warrant therapy with appropriate antibiotics before initiation of any surgical procedure to remove the stone (10). Extracorporeal shock wave lithotripsy (ESWL) was introduced into medical practice in the 1980s, and since then has become one of the main treatment options in patients with renal and/or ureteral calculi (11). Lithotripters have been developed with new sources for generating shock waves, such as electromagnetic and piezoelectric sources, Regarding the Piezoelectric type is a spherical dish is covered with about 3000 small ceramic elements, each of which expands rapidly when a high voltage is applied across them, this rapid 
expansion generates a shock wave (11). The European Association of Urology (EAU), in its urolithiasis guidelines, recommends ESWL as the preferred first-line therapy for all kidney stones smaller than $10 \mathrm{~mm}$. Stones $1 \mathrm{~cm}$ or less in diameter, ESWL achieves stone-free rates of approximately $50 \%$ to $90 \%$ and effectiveness quotients of approximately $50 \%$ to $70 \%$. Shock wave lithotripsy treatment success rates exceeding $70 \%$ have been reported for stones in the upper (71.8\%) and middle (76.5\%) calyces, lower pole stone clearance rates range lower, between $37 \%$ and $61 \%{ }^{(12)}$. In patients with pre-SWL sterile urine, the procedure poses a risk of post-operative UTIs up to $14 \%$ in patients without prophylactic antibiotics (13). Pre-ESWL prophylactic antibiotics have an important role in reducing post-SWL infections, however, previous studies reported conflicting results. The issue of administering prophylactic antibiotics remains controversial in patients with sterile urine undergoing ESWL (14).

The European Association of Urology (EAU) and the American Urological Association (AUA) guidelines proposed different protocols for prophylaxis. The AUA Best Practice Statement on Urological Surgery Antimicrobial Prophylaxis recommends routine antibiotic prophylaxis (15). Conversely, the more published guidelines on urological infections by the EAU advocate for prophylaxis only in patients with urinary drainage tubes, ureteral stents or infected stones ${ }^{(16)}$.

The purpose of this study was to evaluate possible risk factors for post ESWL bacteriuria and consequently to identify patients with higher danger for UTI or sepsis

\section{Methods}

Urine samples from 50 patients underwent ESWL, were collected by clean catch midstream urine collection method in sterile containers and promptly transported to the laboratory. The relevant data pertaining to history, examination and laboratory work up were recorded in predesigned forma, all the samples were subjected to urine culture. Those patients were attending and admitting to AlImamein Al-Kadhimein Medical City conducted with Al-Nahrain University College of Medicine during the period from October 2016 to January 2017. All patients had a urine culture without targeted Antibiotics performed before and after shock wave lithotripsy Loopful of the sample were inoculated on a blood agar and MacConkey agar aerobically for 18-24 hours at $37{ }^{\circ} \mathrm{C}$. The identification of Enterobacteria family was performed according to the biochemical tests indicated in the scheme of Farmer and his co-workers (17). Other bacterial species were identified by Gram stain and biochemical test related to each isolate. Statistical analysis was performed with Epi-Info 7 and Excel programs. Statistical significance was evaluated using the Fisher's exact test with $p<0.05$ considered statistically significant.

\section{Results}

A total of 50 patients who underwent ESWL during the 2-months study period were enrolled in the study. Thirty-three 33 (66\%) out of 50 were Men and 17 (34\%) out of 50 were women with the mean \pm SD age of $45 \pm 14$ years with a ratio of 1.9:1 (Table 1 and Figure 1 and 2). Out of 50 patients suffering were enrolled in this study, the Following patients were with difference underlying diseases as: 10 (20\%) had hypertension and 5 (10\%) had Diabetes mellitus. Out of 50 patients enrolled in the study, 41 (82\%) patients had a renal stone, those patients were grouped according to the site of stone into three anatomical groups that 21 (42\%) right sided renal stone, 20 (40\%) left sided renal stone and $9(18 \%)$ had a ureteral stone with only 5 of 9 patients underwent ESWL with previously placed Double-J stent. The size of treated stone was $(0.64 \mathrm{~cm} \pm 0.48)$ (Table 1). Pre- ESWL results revealed 14 (28\%) were urine culture positive (Table 2), while 23 cases were Post-SWL urine culture positive Table (3).

Proteus microorganism is positive in both patients with history of previous UTI and history of previous surgery (Figures 3, 4 and 5). 
Table 1. Demographic information of the patients

\begin{tabular}{|c|c|c|}
\hline Age (years) & Frequency & Percent (\%) \\
\hline $10-19$ & 1 & 2 \\
\hline $20-29$ & 5 & 10 \\
\hline $30-39$ & 13 & 26 \\
\hline $40-49$ & 17 & 34 \\
\hline $50-59$ & 8 & 16 \\
\hline $60-69$ & 3 & 6 \\
\hline 70-79 & 3 & 6 \\
\hline Total & 50 & 100 \\
\hline \multicolumn{3}{|l|}{ Gender } \\
\hline Male & 33 & 66 \\
\hline Female & 17 & 34 \\
\hline Total & 50 & 100 \\
\hline \multicolumn{3}{|c|}{ The site and side of Renal stone } \\
\hline Right & 21 & 42 \\
\hline Left & 20 & 40 \\
\hline Ureter & 9 & 18 \\
\hline Total & 50 & 100 \\
\hline \multicolumn{3}{|c|}{ The size of Renal stone } \\
\hline$<1 \mathrm{~cm}$ & 18 & 36 \\
\hline$>1 \mathrm{~cm}$ & 32 & 64 \\
\hline Total & 50 & 100 \\
\hline \multicolumn{3}{|c|}{ History of Urinary tract infection } \\
\hline Yes & 32 & 76 \\
\hline No & 18 & 24 \\
\hline Total & 50 & 100 \\
\hline \multicolumn{3}{|c|}{ History of previous surgery } \\
\hline Yes & 23 & 46 \\
\hline No & 27 & 54 \\
\hline Total & 50 & 100 \\
\hline
\end{tabular}

Table 2. Demographic information of the patients

\begin{tabular}{ccc}
\hline Pre-ESWL urine culture Results & Frequency & Percent (\%) \\
\hline Negative culture & 36 & 72 \\
Bacillus & 1 & 2 \\
Corynbacterium & 1 & 2 \\
E.Coli & 4 & 8 \\
Enterococcus & 2 & 4 \\
Klebsiella & 1 & 2 \\
Proteus & 2 & 4 \\
Staphylococcus Aureus & 1 & 2 \\
Streptococcus Agalactiae & 2 & 4 \\
\hline
\end{tabular}


Table 3. The results of Post-ESWL urine culture

\begin{tabular}{ccc}
\hline Post-ESWL urine culture Results & Frequency & Percent (\%) \\
\hline Negative culture & 27 & 54 \\
Bacillus & 1 & 2 \\
Corynebacterium & 1 & 2 \\
E. coli & 4 & 8 \\
Enterococcus & 3 & 6 \\
Klebsiella & 2 & 4 \\
Proteus & 7 & 14 \\
Staphylococcus Aureus & 5 & 10 \\
\hline
\end{tabular}

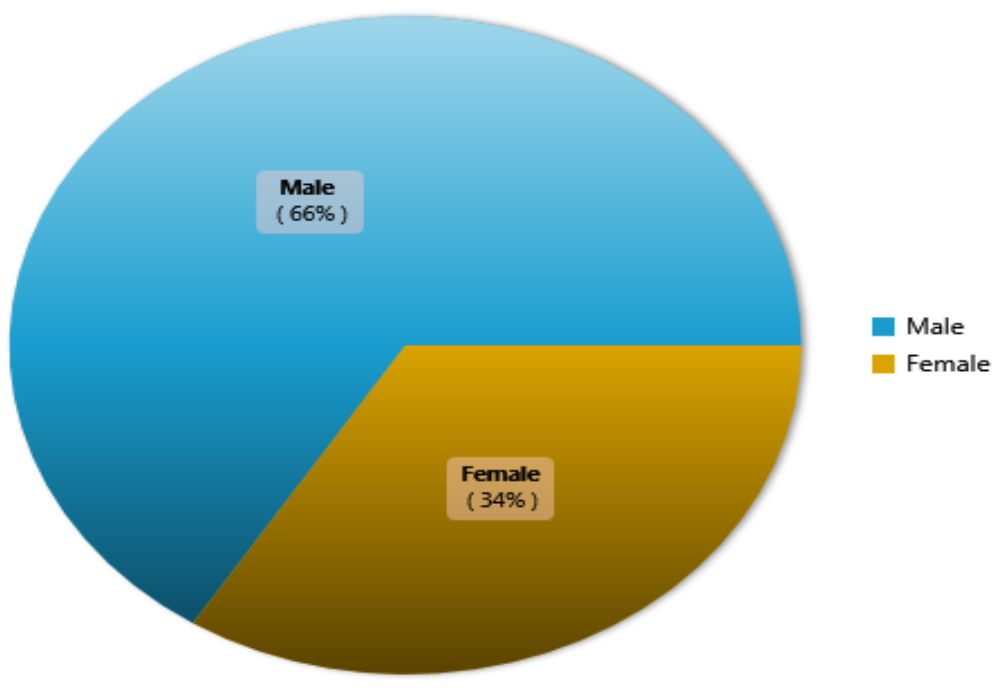

Figure 1. Sex distribution among patients of the study

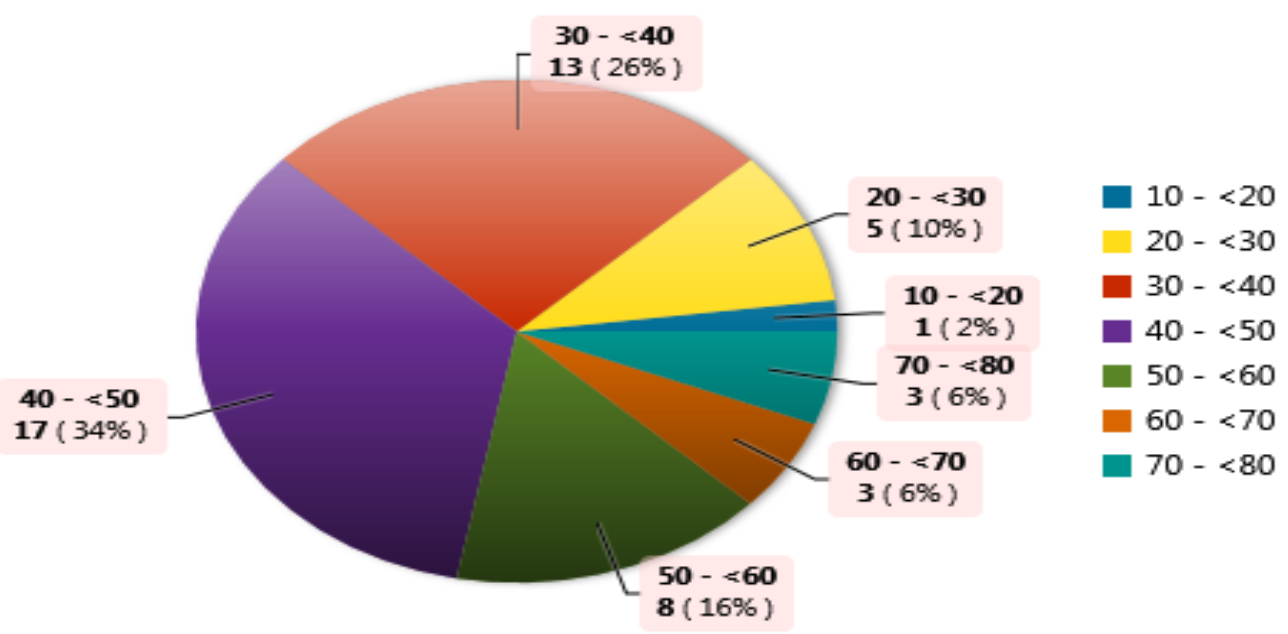

Figure 2. Age distribution among patients of the study 




Figure 3. The prevalence of patients with history of UTI

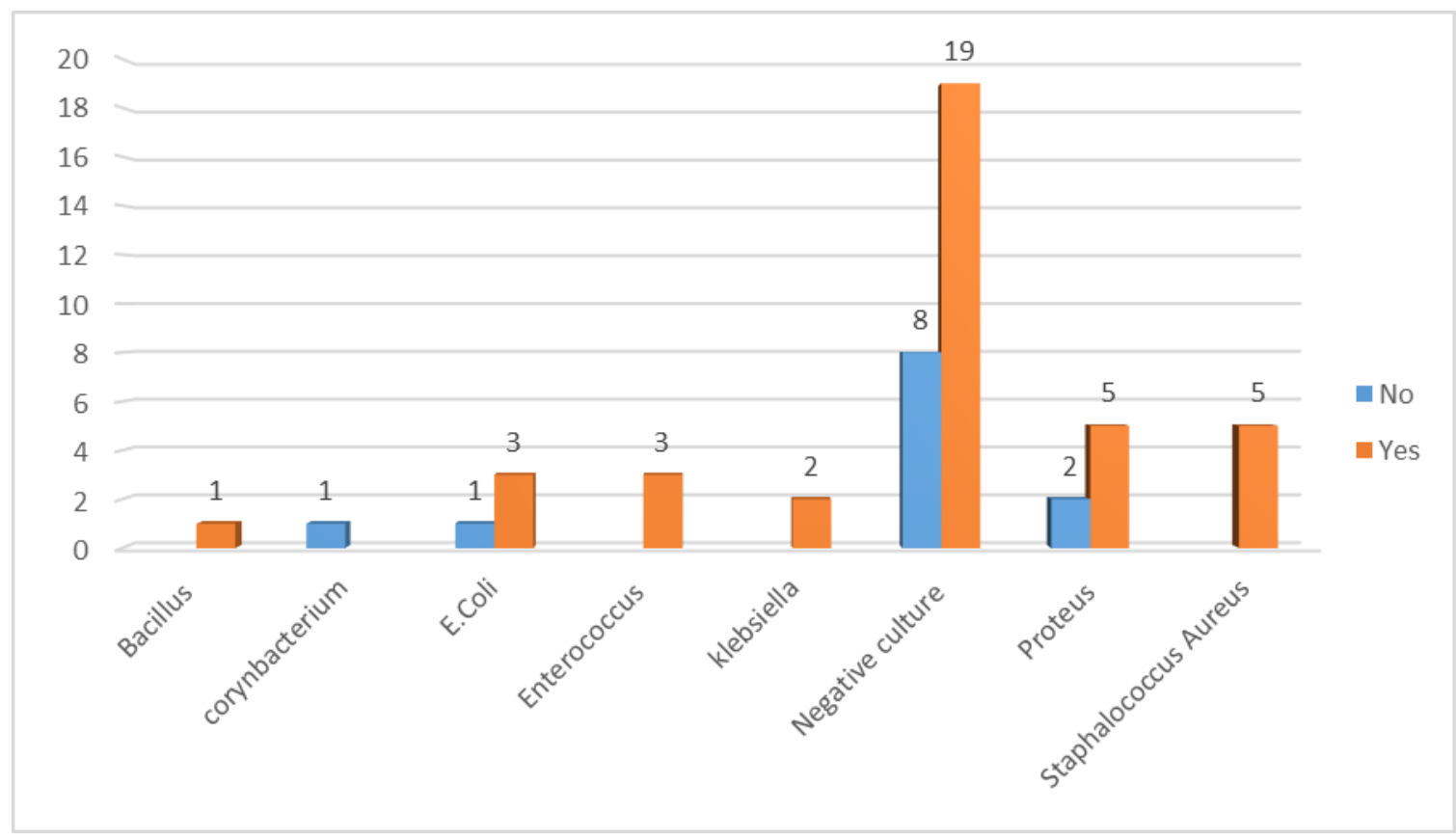

Figure 4. The prevalence of Post ESWL urine culture with the history of UTI 


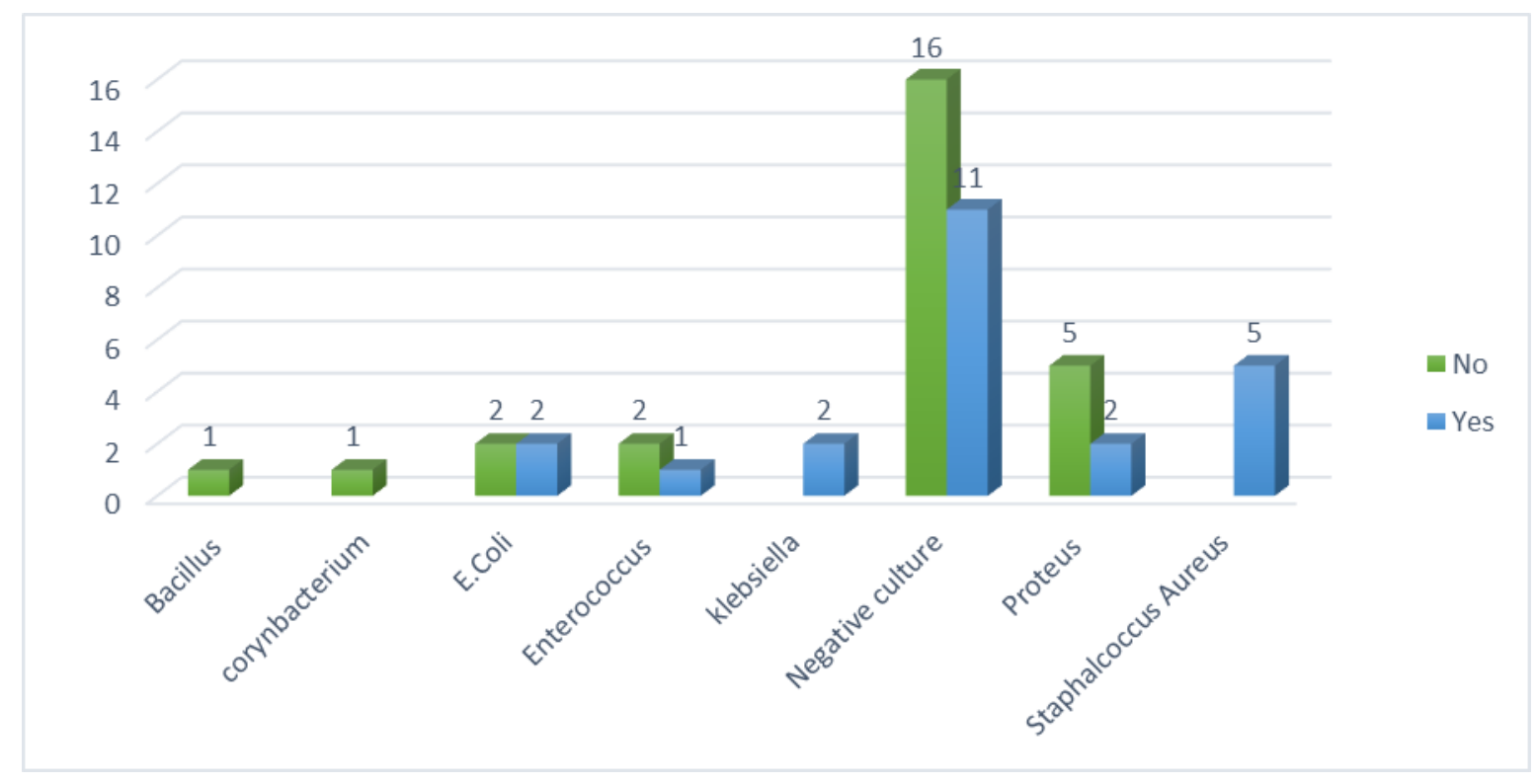

\section{Figure 5. The prevalence of Post ESWL urine culture with history of previous surgery}

\section{Discussion}

Clearly controversy exists regarding the need for antibiotic prophylaxis with ESWL as underlined by the differences in the AUA and EAU guidelines for antimicrobial prophylaxis. The AUA Best Practice Policy Statement on Urological Surgery Antimicrobial Prophylaxis states that antibiotic prophylaxis is indicated in all patients with a duration of therapy of 24 hours or less. This recommendation is based on a meta-analysis by Pearle and Roehrborn evaluating 8 randomized controlled trials and 6 clinical trials ${ }^{(14)}$, their meta-analysis of these 8 RCTs demonstrated a reduction in the median probability of UTI after SWL from $5.7 \%$ in the no treatment arms to $2.1 \%$ in the antibiotic treatment arms. However, there are several key limitations with this meta-analysis and the RCTs that comprise it. Alternatively, the EAU Guidelines on Urological Infections only recommend prophylaxis in cases of "internal stent and treatment, due to the increased bacterial burden (e.g. indwelling catheter, nephrostomy tube, or infectious stones)" (18). In this prospective single cohort study of 50 patients with Pre-\& after ESWL urine culture without receiving antibiotics prophylaxis; in this research analyzed risk factors that could be related to a positive urine culture. The factors considered were gender, age, diabetes mellitus, arterial hypertension, and history of previous surgery (including Double $J$ stent and Nephrostomy tube), personal history of UTI, stone size and stone location; Found that the patients having history of UTI and history of previous surgery with determining the patients had a history of Double $J$ tube in case of ureteral stone and nephrostomy tube as a part of previous surgery is significant according to the statistical analysis of this study $(P=0.04)$; this study support the EAU guidelines that no routine prophylaxis is necessary for patients undergoing ESWL with sterile urine cultures. However, that the patients with a history of UTI and a history of previous surgery (with ureteral stent or nephrostomy tube) recommended antibiotic prophylaxis prior to ESWL. Further supporting the notion that antibiotic prophylaxis is not required in all patients undergoing ESWL. In this prospective study revealed that the gender, age, number of stone attacks, the size of stone was an independent risk factor for significant urine culture. This current study had some limitations, that had no comparison group with prophylactic antibiotic treatment. It would 
have been useful to determine whether the rates of bacteriuria and symptomatic urinary tract infection were or were not similar. Moreover, this study could not determine whether the presence of a Double-J stent before ESWL is an independent risk factor for bacteriuria after ESWL, although this is considered a risk factor by the AUA and EAU $(13,14)$. Perhaps it should increase the population to determine that fact. Another limitation is that it could not determine the risk factors associated with symptomatic infections and serious events. This analysis was not possible due to the low incidence of these events. Accordingly, future studies are needed to clarify the appropriate indications for targeted antibiotic prophylaxis before ESWL with Pre-\& Post ESWL urine culture, and the ideal choice and duration of antibiotic treatment.

This prospective cohort study revealed the need for universal antibiotic prophylaxis before ESWL as UTIs and ASB are low incidence in patients with negative urine cultures treated with targeted antibiotic Prophylaxis. This suggested that prophylactic antibiotics in these patients is unnecessary with no benefit in reducing infectious complications and may pose the risk of increased bacterial resistance and side effects of antibiotics. This led us to conclude that antibiotic prophylaxis is not justified without defined risk factors such as positive urine culture before ESWL, an external bladder catheter or nephrostomy tube and a history of infectious stones or recurrent urinary tract infection.

\section{Acknowledgments}

First, authors would like to thank to all patients that participated in the study. In addition, authors thank medical staff of ESWL department in the Al-Imamein Al-Kadhimein Medical City, Baghdad. Special thanks to Microbiology department of Collage of Medicine, Al-Nahrain University.

\section{Authors contribution}

Dr. Khaleel drafting the article, revising it critically for important intellectual content and made collection of urine sample from the patients Pre-\& Post ESWL. Dr. Hassan made a conventional method of urine culture in Microbiology laboratory in the Collage of Medicine, Al-Nahrain university. Dr. Al-Anbary made a statistical analysis of the research and give a final approval of the version of the research to be submitted.

\section{Conflict of interest}

Authors declare no conflict of interest.

\section{Funding}

There was no funding source for this research.

\section{References}

1. Stamatelou KK, Francis ME, Jones A, et al. Time trends in reported prevalence of kidney stones in the United States: 1976-1994. Kidney Int. 2003; 63(5): 1817-23. doi: 10.1046/j.1523-1755.2003. 00917.x

2. Song L, Maalouf NM. 24-Hour urine calcium in the evaluation and management of nephrolithiasis. JAMA. 2017; 318(5): 474-5. doi: 10.1001/jama.2017.7085.

3. Marien T, Miller NL. Treatment of the Infected Stone: Urol Clin North Am. 2015; 42(4): 459-72. doi: 10.1016/j.ucl.2015.05.009.

4. Bichler $\mathrm{KH}$, Eipper $\mathrm{E}, \mathrm{Naber} \mathrm{K}$, et al. Urinary infection stones. Int J Antimicr Agents. 2002; 19(6): 488-98. doi: 10.1016/S0924-8579(02)00088-2.

5. Dason S, Dason JT, Kapoor A. Guidelines for the diagnosis and management of recurrent urinary tract infection in women. Can Urol Assoc J. 2011; 5(5): 316-22. doi: 10.5489/cuaj.11214.

6. Gault MH, Longerich LL, Crane G, et al. Bacteriology of Urinary Tract Stones. J Urol. 1995; 153(4): 116470. doi: 10.1016/S0022-5347(01)67539-6.

7. Kajender EO, Çiftçioglu N. Nano bacteria: an alternative mechanism for pathogenic intra and extracellular calcification and stone formation. Proc Natl Acad Sci USA. 1998; 95(14): 8274-9. doi: 10.1073/pnas.95.14.8274.

8. Howell MD, Davis AM. Management of sepsis and Septic Shock. JAMA. 2017; 317(8): 847-8. doi: 10.1001/jama.2017.0131.

9. Korets R, Graversen JA, Kates $M$, et al. Postpercutaneous nephrolithotomy systemic inflammatory response: a prospective analysis of preoperative urine, renal pelvic urine and stone cultures. J Urol. 2011; 186(5): 1899-903. doi: 10.1016/j.juro.2011.06.064.

10. Micali S, Sighinolfi MC, Grande M, et al. Dornier lithotripter S 220 F EMSE: the first report of over 1000 treatments. Urology. 2009; 74(6): 1211-4. doi: 0.1016/j.urology.2009.05.101.

11. Saw KC, Lingeman JE. Management of calyceal stones. AUA Update Series. 1999; 20: 154-9.

12. Türk C, Petřík A, Sarica K3, et al. EAU Guidelines on Interventional Treatment for Urolithiasis. Eur Urol. 
2016;

69(3):

475-82.

doi:

10.1016/j.eururo.2015.07.041.

13. Lu $\mathrm{Y}$, Tianyong $\mathrm{F}$, Ping $\mathrm{H}$, et al. Antibiotic prophylaxis for shock wave lithotripsy in patients with sterile urine before treatment may be unnecessary: A systematic review and meta-analysis. Journal of Urology. 2012; 188(2): 441-8. doi: 10.1016/j.juro.2012.04.014.

14. Pearle MS, Roehrborn CG. Antimicrobial prophylaxis prior to shock wave lithotripsy in patients with sterile urine before treatment: A meta-analysis and cost effectiveness analysis. Urology. 1997; 49(5): 679-86. doi: 10.1016/S0090-4295(96)00626-7.

15. Wolf JS, Jr, Bennett CJ, Dmochowski RR, et al. Best practice policy statement on urologic surgery antimicrobial prophylaxis. J Urol. 2008; 179(4): 137990. doi: 10.1016/j.juro.2008.01.068
16. Grabe $M$, Bishop $M C$, Bjerklund-Johansen $T E$, et al. Guidelines on the management of urinary and male genital tract infections. Arnhem, the Netherlands: European Association of Urology; 2008.

17. Farmer JJ, Davis RB, and Hickman-Brenner FW, Et al. Biochemical identification of new species and biotypes of Enterobacteriaceae isolated from clinical specimens. J Clin Microbiol. 1985; 21(1): 46-76.

18. Dinçel $C$, Ozdiler $E$, Ozenci $H$ et al. Incidence of urinary tract infection in patients without bacteriuria undergoing SWL: comparison of stone types. J Endourol. 1998; 12(1): 1-3.

Correspondence to Dr. Ahmed A. Khaleel E-mail: Abk19933@gmail.com Received Oct. 26 2017 Accepted Feb. $26^{\text {th }} 2018$ 